\title{
Indice de Morse des applications $p$-harmoniques
}

par

\author{
Ahmad EL SOUFI et Albert JEUNE \\ Laboratoire de Mathématiques, \\ Université de Tours, Parc de Grandmont, \\ 37200 Tours, France.
}

RÉSUMÉ. - Dans cet article, nous nous intéressons à l'estimation et au calcul de l'indice de Morse des applications $p$-harmoniques, où $p \in[2,+\infty[$. Nous y traitons notamment le cas de l'application identité, ainsi que celui des applications définies sur des sphères où à valeurs dans des sphères. Les résultats que nous obtenons généralisent un grand nombre de ceux obtenus pour les applications harmoniques $(p=2)$ dans [2], [3] et [9].

ABSTRACT. - In this paper, we investigate estimates and calculation of the Morse index of $p$-harmonic maps, with $p \in[2,+\infty[$. More particularly, we deal with the identity map and maps from or to spheres. Our results extend those obtained for harmonic maps (case $p=2$ ) in [2], [3] and [9].

\section{INTRODUCTION}

Dans cet article nous nous intéressons aux points critiques, dits applications $p$-harmoniques, de la fonctionnelle $p$-énergie $E_{p}, p \in$ $[2,+\infty[$, définie sur l'espace des applications différentiables d'une variété riemannienne compacte $(M, g)$ à valeur dans une variété riemannienne

Ce travail a été partiellement soutenu par le contrat CEE GADGET SC1-0105-C. 
$\left(N, g^{\prime}\right)$. Cette notion d'application $p$-harmonique est une généralisation naturelle de celle bien connue d'application harmonique qui correspond ici au cas $p=2$.

Une bonne partie des résultats de cet article porte sur l'indice de Morse des applications $p$-harmoniques. Cet invariant, que l'on note $\operatorname{Ind}_{p}(\phi)$ pour une application $p$-harmonique $\phi$, mesure en fait le degré d'instabilité du point critique $\phi$. L'application $\phi$ est dite $p$-stable si $\operatorname{Ind}_{p}(\phi)=0$.

Les premiers résultats concernant la stabilité et l'indice de Morse des applications harmoniques ont été obtenus par Mazet [9] et Smith [11]. Le cas particulier de l'application identité fut largement étudié par ces auteurs. Au paragraphe II du présent article nous nous intéressons également à ce cas et nous montrons (théorème 1) que, si $(M, g)$ est une variété compacte orientable de dimension $m$, alors, pour tout $p \geq m$, l'identité $I_{M}$ de $M$ minimise la $p$-énergie $E_{p}$ sur l'ensemble des applications de degré non nul de $M$ dans $M$. Nous en déduisons que $I_{M}$ est $p$-stable pour tout $p \geq m$.

Dans le cas $p<m=\operatorname{dim} M$, nous généralisons au cas $p$-harmonique les arguments de Smith pour montrer que (théorèmes 2 et 3 ) :

(i) $\operatorname{Ind}_{p}\left(I_{M}\right)$ est minoré par la codimension de l'espace des champs de Killing dans celui des champs conformes de $(M, g)$.

(ii) Si $(M, g)$ est d'Einstein, alors $\operatorname{Ind}_{p}\left(I_{M}\right)$ est égal au nombre de valeurs propres de $(M, g)$ que contient l'intervalle $] 0,2 m c /(m+p-2)[$, où $m c$ est la courbure scalaire de $(M, g)$.

Une classe particulière d'applications $p$-harmoniques est constituée par les immersions minimales homothétiques. Au paragraphe III de cet article nous montrons (théorème 4) que, si $\phi:(M, g) \rightarrow\left(N, g^{\prime}\right)$ est une telle application, alors

$$
\operatorname{Ind}_{p}(\phi) \geq \operatorname{Ind}_{p}\left(I_{M}\right),
$$

et que, si $\phi$ est totalement géodésique, alors

$$
\operatorname{Ind}_{p}(\phi)-\operatorname{Ind}_{V}(\phi)=\operatorname{Ind}_{p}\left(I_{M}\right),
$$

où $\operatorname{Ind}_{V}(\phi)$ est l'indice de $\phi$ pour la fonctionnelle Volume.

Ces résultats permettent le calcul de l'indice d'un nombre non négligeable d'applications $p$-harmoniques dont l'injection canonique $I_{m, n}$ de la sphère canonique $\mathbb{S}^{m}$ de dimension $m$ dans $\mathbb{S}^{n}$, et l'immersion canonique $j_{m}$ de $\mathbb{S}^{m}$ dans l'espace projectif complexe $\mathbb{C} P^{m}$ ( $c f$. corollaires 2 et 3 ).

Le paragraphe IV est consacré au cas où la variété source est la sphère canonique $\mathbb{S}^{m}$. Rappelons que Xin [13] avait montré que, pour toute application harmonique $\phi$ de $\mathbb{S}^{m}, m \geq 3$, dans une variété $\left(N, g^{\prime}\right)$, on 
a $\operatorname{Ind}_{2}(\phi) \geq 1$. Ce résultat fut amélioré par le premier auteur dans [3] où l'on trouve la minoration optimale : $\operatorname{Ind}_{2}(\phi) \geq m+1$. Aussi bien dans [13] que dans [3], l'idée de base est de calculer la variation seconde de l'énergie dans la direction des champs du type $d \phi(V)$, où $V$ est un champ conforme de $\mathbb{S}^{m}$. Cependant, les deux démarches sont différentes. En effet, la preuve de Xin est fondée sur la formule générale de la variation seconde, alors que le résultat de [3] découle du calcul de la variation première en tout point, le long du groupe à un paramètre engendré par le champ conforme $V$.

Dans le cas de la $p$-énergie et des applications $p$-harmoniques, la formule générale de la variation seconde ( $c f$. (3)) devient très peu maniable, ce qui rend très difficile la généralisation des arguments de Xin. Par contre, la méthode développée par le premier auteur dans [2] et [3] présente l'avantage de s'étendre de manière naturelle à ce type de fonctionnelle et permet d'obtenir en particulier la minoration optimale suivante (théorème 5) :

$$
\operatorname{Ind}_{p}(\phi) \geq m+1
$$

valable pour toute application $p$-harmonique non constante $\phi$, avec $p<m$, de $\mathbb{S}^{m}$ dans $\left(N, g^{\prime}\right)$.

Cette méthode présente un deuxième avantage. En effet, outre le calcul de la variation seconde dans la direction des champs conformes et la minoration de l'indice, elle permet d'avoir le résultat global suivant (théorème 6) : si $G(m)$ est le groupe des difféomorphismes conformes de $\mathbb{S}^{m}$, alors toute application $p$-harmonique $\phi: \mathbb{S}^{m} \rightarrow\left(N, g^{\prime}\right)$ vérifie

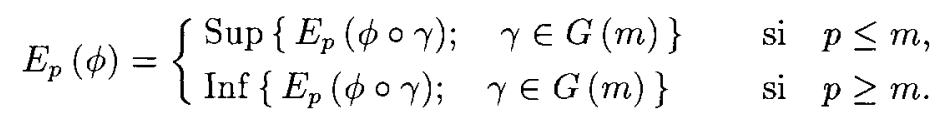

De plus, si $\phi$ est non constante et $p \neq m$, alors l'égalité $E_{p}(\phi \circ \gamma)=E_{p}(\phi)$ n'a lieu que si $\gamma$ est une isométrie.

Le dernier paragraphe traite du cas où la variété but est une sphère. Nous y faisons une étude parallèle à celle développée au paragraphe IV. Nous y montrons en particulier que toute application $p$-harmonique non constante $\phi:(M, g) \rightarrow \mathbb{S}^{n}$ vérifie (théorème 7$)$ :

$$
\operatorname{Ind}_{p}(\phi) \geq n-p \text {. }
$$

De plus, sous une hypothèse de positivité sur le tenseur de $p$-énergieimpulsion de $\phi$, nous montrons que (théorèmes 8 et 9 ) :

$$
\operatorname{Ind}_{p}(\phi) \geq n+1
$$

Vol. $13, n^{\circ} 2-1996$. 
et

$$
E_{p}(\phi)=\operatorname{Sup}\left\{E_{p}(\gamma \circ \phi) ; \quad \gamma \in G(n)\right\}
$$

Ces résultats sont une généralisation de ceux obtenus, pour $p=2$, par le premier auteur dans [2]. Noter que le cas des applications harmoniques à valeurs dans une sphère avait d'abord été étudié par Leung [8].

\section{PRÉLIMINAIRES}

Dans toute la suite de cet article, on désignera par $(M, g)$ et $\left(N, g^{\prime}\right)$ deux variétés riemanniennes connexes de dimensions respectives $m$ et $n$. La variété $M$ sera supposée compacte. Les produits scalaires associés à $g$ et $g^{\prime}$ seront tous notés $\langle$,$\rangle .$

Soit $\phi$ une application différentiable de $M$ dans $N$. Pour tout réel $p \geq 2$, on appelle $p$-énergie de $\phi$ la quantité :

$$
E_{p}(\phi)=\frac{1}{p} \int_{M}|d \phi|^{p} d v
$$

où $d v$ est l'élément de volume riemannien associé à $g$, et où $|d \phi|$ est la norme de Hilbert-Schmidt de $d \phi$ : si $\sigma$ et $\sigma^{\prime}$ sont deux 1-formes sur $M$ à valeurs dans $\phi^{*} T N$, alors,

$$
\left\langle\sigma, \sigma^{\prime}\right\rangle(x)=\sum_{i=1}^{m} g^{\prime}\left(\sigma\left(e_{i}\right), \sigma^{\prime}\left(e_{i}\right)\right)
$$

$\left\{e_{i}\right\}_{i}$ étant une base $g$-orthonormée de $T_{x} M$.

Soit $\Gamma(\phi)$ l'espace des champs de vecteurs le long de $\phi$, i.e. les sections du fibré $\phi^{*} T N$. On désigne respectivement par $D$ et $\nabla$ les connexions canoniques de $(M, g)$ et $\left(N, g^{\prime}\right)$, et par $\nabla^{\phi}$ la connexion induite sur $\phi^{*} T N$ par $\nabla$.

La variation première de la $p$-énergie en $\phi$ dans la direction d'un champ $V \in \Gamma(\phi)$ est donnée par la formule :

$$
\left(D E_{p}\right)_{\phi}(V)=\left.\frac{d}{d t} E_{p}\left(\phi_{t}\right)\right|_{t=0}=-\int_{M}|d \phi|^{p-2}\left\langle d \phi(\cdot), \nabla^{\phi} V\right\rangle d v
$$

où $\left(\phi_{t}\right)_{t}$ est une famille d'applications différentiables telle que $\phi_{0}=\phi$ et $\left.\frac{d}{d t} \phi_{t}\right|_{t=0}=V$. L'application $\phi$ sera dite $p$-harmonique si $\left(D E_{p}\right)_{\phi}(V)=0$ pour tout $V \in \Gamma(\phi)$. Le cas $p=2$ correspond à celui où $\phi$ est harmonique. 
L'extension naturelle de $\nabla^{\phi}$ aux $k$-formes $\sigma$ à valeurs dans $\phi^{*} T N$ est donnée par:

$$
\begin{aligned}
\left(\nabla_{X}^{\phi} \sigma\right)\left(X_{1}, \ldots, X_{k}\right)= & \nabla_{X}^{\phi}\left(\sigma\left(X_{1}, \ldots, X_{k}\right)\right) \\
& -\sum_{i=1}^{k} \sigma\left(X_{1}, \ldots, D_{X} X_{i}, \ldots, X_{k}\right)
\end{aligned}
$$

On appelle $p$-tension de $\phi$ le champ $\tau_{p}(\phi)$ donné par :

(1) $\tau_{p}(\phi)=\operatorname{tr}_{g} \nabla^{\phi}\left(|d \phi|^{p-2} d \phi\right)(\cdot)=|d \phi|^{p-2} \tau_{2}(\phi)+d \phi\left(\nabla|d \phi|^{p-2}\right)$

où $\nabla|d \phi|^{p-2}$ désigne le gradient de $|d \phi|^{p-2}$ dans $(M, g)$. Après une intégration par parties la formule de la variation première ci-dessus devient :

$$
\left(D E_{p}\right)_{\phi}(V)=-\int_{M}\left\langle\tau_{p}(\phi), V\right\rangle d v
$$

Il en découle que $\phi$ est $p$-harmonique si et seulement si $\tau_{p}(\phi)=0$. Notons que d'après (1), si $|d \phi|$ est constante, alors, pour tout $p \geq 2$, $\phi$ est $p$-harmonique si et seulement si elle est harmonique.

Soit $R^{N}$ le tenseur de courbure de $\left(N, g^{\prime}\right)$. On pose, pour tous $V$, $W \in \Gamma(\phi)$,

$$
\operatorname{Ric}^{\phi}(V, W)=\operatorname{tr}_{g} R^{N}(d \phi(\cdot), V, d \phi(\cdot), W) .
$$

La variation seconde de $E_{p}$ en $\phi p$-harmonique dans la direction de $V$ et $W$ est alors donnée par (voir par exemple [7]) :

$$
\text { (3) } \begin{aligned}
H_{p}^{\phi}(V, W)= & \left.\frac{\partial^{2}}{\partial s \partial t} E_{p}\left(\phi_{s, t}\right)\right|_{(0,0)} \\
= & \int_{M}|d \phi|^{p-2}\left(\operatorname{tr}_{g}\left\langle\nabla^{\phi} V, \nabla^{\phi} W\right\rangle-\operatorname{Ric}^{\phi}(V, W)\right) d v \\
& +(p-2) \int_{M}|d \phi|^{p-4}\left\langle\nabla^{\phi} V, d \phi(\cdot)\right\rangle\left\langle\nabla^{\phi} W, d \phi(\cdot)\right\rangle d v
\end{aligned}
$$

On appelle $p$-indice de $\phi$, et on le note $\operatorname{Ind}_{p}(\phi)$, la dimension des sousespaces maximaux de $\Gamma(\phi)$ sur lesquels la forme quadratique $H_{p}^{\phi}$ est définie négative, i.e. 
$\operatorname{Ind}_{p}(\phi)=\operatorname{Sup}\left\{\operatorname{dim} E ; E \subset \Gamma(\phi)\right.$ t.q. $H_{p}^{\phi}$ est définie négative sur $\left.E\right\}$. L'application $\phi$ sera dite $p$-stable si $\operatorname{Ind}_{p}(\phi)=0$.

Nous déduisons directement de la formule (3) les propositions suivantes :

Proposition 1. - Soit $\left(N, g^{\prime}\right)$ une variété à courbure sectionnelle négative ou nulle. Alors toute application p-harmonique, $p \geq 2$, d'une variété riemannienne compacte $(M, g)$ dans $\left(N, g^{\prime}\right)$ est p-stable.

Dans le cas particulier des applications harmoniques à densité d'énergie constante, on a la

PROPOSITION 2. - Soit $\phi$ une application harmonique d'une variété compacte $(M, g)$ dans une variété $\left(N, g^{\prime}\right)$. Si $|d \phi|$ est constante, alors, pour tout $p \geq 2$, $\phi$ est $p$-harmonique et l'application $p \rightarrow \operatorname{Ind}_{p}(\phi)$ est décroissante sur $[2,+\infty[$.

Preuve. - Soient $p \geq q \geq 2$ deux réels. Comme $|d \phi|$ est constante, la formule (3) nous donne pour tout $V \in \Gamma(\phi)$,

$$
\begin{aligned}
|d \phi|^{p-q} H_{q}^{\phi}(V, V)= & H_{p}^{\phi}(V, V)-(p-q)|d \phi|^{p-4} \\
& \times \int_{M}\left\langle\nabla^{\phi} V, d \phi(\cdot)\right\rangle^{2} d v
\end{aligned}
$$

Le résultat en découle immédiatement.

\section{INDICE DE L'IDENTITÉ}

Dans ce paragraphe nous allons nous intéresser à l'application identité $I$ de $(M, g)$ qui est évidemment $p$-harmonique pour tout $p$.

Pour $p \geq m$, nous avons le

THÉORÈME 1. - Soit $(M, g)$ une variété riemannienne compacte orientable de dimension $m$ et soit $p \in[m,+\infty[$. Pour toute application $\phi: M \rightarrow M$ de degré non nul on a

$$
E_{p}(\phi) \geq E_{p}(I),
$$

où, pour $p>m($ resp. $p=m)$, l'égalité a lieu si et seulement si $\phi$ est une isométrie (resp. une transformation conforme) de $(M, g)$. En particulier, I est p-stable pour tout $p \geq m$. 
Preuve. - D'après [12], si l'on note $V$ la fonctionnelle Volume, on a

$$
E_{m}(\phi) \geq m^{(m-2) / 2} V(\phi),
$$

où l'égalité a lieu si et seulement si $\phi$ est conforme, i.e., $\phi^{*} g=\frac{|d \phi|^{2}}{m} g$. Or, on a $V(\phi)=|\operatorname{deg} \phi| V(M)$. D'où

$$
E_{m}(\phi) \geq m^{(m-2) / 2}|\operatorname{deg} \phi| V(M)=|\operatorname{deg} \phi| E_{m}(I)
$$

car $|d I|^{2}=m$.

Pour tout $p \geq m$ on a (inégalité de Hölder) :

$$
E_{p}(\phi) \geq \frac{m^{p / m}}{p} V(M)^{1-p / m} E_{m}(\phi)^{p / m}
$$

où, si $p>m$, l'égalité a lieu si et seulement si $|d \phi|^{2}$ est constante. D'où

$$
\begin{aligned}
E_{p}(\phi) & \geq \frac{m^{p / m}}{p} V(M)^{1-p / m}|\operatorname{deg} \phi|^{p / m} E_{m}(I)^{p / m} \\
& =\frac{m^{p / 2}}{p}|\operatorname{deg} \phi|^{p / m} V(M)=|\operatorname{deg} \phi|^{p / m} E_{p}(I) \geq E_{p}(I) .
\end{aligned}
$$

L'égalité $E_{p}(\phi)=E_{p}(I)$ entraine donc que $\phi$ est conforme de degré \pm 1 et, si $p>m$, que $|d \phi|^{2}$ est constante égale à $m$. On en déduit immédiatement le résultat.

La formule de la variation seconde devient dans le cas $\phi=I$ :

$$
\begin{aligned}
& H_{p}^{I}(V, W)=m^{(p-2) / 2} \int_{M}(\langle D . V, D . W\rangle \\
& \left.\quad-\operatorname{Ric}^{M}(V, W)\right) d v+(p-2) m^{(p-4) / 2} \int_{M} \delta V \delta W d v
\end{aligned}
$$

où $\operatorname{Ric}^{M}$ est la courbure de Ricci de $(M, g)$ et où $\delta V$ est la divergence de $V$. Ici $V$ et $W$ sont des champs de vecteurs sur $M$. Les formules intégrales classiques donnent ( $c f$. par exemple [14]) :

$$
\int_{M}\left(\langle D . V, D . V\rangle-\operatorname{Ric}^{M}(V, V)\right) d v=\int_{M}\left(\frac{1}{2}\left|L_{V} g\right|^{2}-(\delta V)^{2}\right) d v
$$

où $L_{V} g$ est la dérivée de Lie de $g$ par rapport à $V$. D'où

$$
\begin{gathered}
\text { (6) } H_{p}^{I}(V, V)=m^{(p-4) / 2} \int_{M}\left(\frac{m}{2}\left|L_{V} g\right|^{2}+(p-2-m)(\delta V)^{2}\right) d v \\
=m^{(p-4) / 2}\left[\frac{m}{2} \int_{M}\left(\left|L_{V} g\right|^{2}-\frac{4}{m}(\delta V)^{2}\right) d v+(p-m) \int_{M}(\delta V)^{2} d V\right] \\
\geq m^{(p-4) / 2}(p-m) \int_{M}(\delta V)^{2} d v
\end{gathered}
$$


En effet, dans une base orthonormée $\left\{e_{i}\right\}$ qui diagonalise $L_{V} g$ en un point $x$ de $M$, on a :

$$
\begin{aligned}
\left|L_{V} g\right|^{2} & =\sum_{i} L_{V} g\left(e_{i}, e_{i}\right)^{2}=4 \sum_{i}\left\langle D_{e_{i}} V, e_{i}\right\rangle^{2} \\
& \leq \frac{4}{m}\left(\sum_{i}\left\langle D_{e_{i}} V, e_{i}\right\rangle\right)^{2}=\frac{4}{m}(\delta V)^{2}
\end{aligned}
$$

De plus, l'égalité $\left|L_{V} g\right|^{2}=\frac{4}{m}(\delta V)^{2}$ entraîne qu'en tout point $x \in M$, les $L_{V} g\left(e_{i}, e_{i}\right)$ sont tous égaux, c'est-à-dire que $L_{V} g$ est proportionnel à $g$ et donc que le champ $V$ est conforme. On en déduit la proposition suivante :

Proposition 3. - Soit $(M, g)$ une variété riemannienne compacte de dimension $m$. Pour tout $p \geq m$, l'identité $I$ de $M$ est $p$-stable et le noyau de $H_{p}^{I}$ est donné par

$$
\operatorname{Ker} H_{p}^{I}=\left\{\begin{array}{lll}
C & \text { si } & p=m \\
K & \text { si } & p>m
\end{array}\right.
$$

où $C$ est l'espace des champs conformes et où $K$ est l'espace des champs de Killing de $(M, g)$.

Preuve. - L'hypothèse $p \geq m$ entraîne d'après (6) qu'on $\mathrm{a}$, pour tout champ de vecteurs $V$ sur $M, H_{p}^{I}(V, V) \geq 0$ et que l'égalité $H_{p}^{I}(V, V)=0$ n'a lieu que si $V$ est conforme et si $(p-m) \delta V=0$. D'où, $I$ est $p$-stable et

$$
\operatorname{Ker} H_{p}^{I} \subset\{V \in C ;(p-m) \delta V=0\}=\left\{\begin{array}{lll}
C & \text { si } & p=m \\
K & \text { si } & p>m
\end{array}\right.
$$

Réciproquement, l'invariance de $E_{p}$ sous l'action du groupe d'isométries (resp. du groupe conforme pour $p=m$ ) entraîne immédiatement qu'on a $\mathrm{K} \subset \operatorname{Ker} H_{p}^{I}$ (resp. $C \subset \operatorname{Ker} H_{m}^{I}$ ). D'où le résultat.

Remarque. - Il est clair, d'après (5), que si $\operatorname{Ric}^{M}$ est non-positif, alors $I$ est $p$-stable pour tout $p \geq 2$.

Une autre conséquence de (6) est le

ThÉORÈME 2. - Soit $(M, g)$ une variété riemannienne compacte de dimension $m$. Pour tout $p \in[2, m[$, on a

$$
\operatorname{Ind}_{p}(I) \geq \operatorname{dim} C / K \text {. }
$$


Preuve. - Pour tout champ conforme $V$ sur $M$ on a

$$
L_{V} g=-\frac{2}{m}(\delta V) g
$$

En remplaçant dans (6) on obtient (avec $p<m$ ) :

$$
H_{p}^{I}(V, V)=(p-m) m^{(p-4) / 2} \int_{M}(\delta V)^{2} d v \leq 0
$$

où l'égalité $H_{p}^{I}(V, V)=0$ a lieu si et seulement si $\delta V=0$, c'est-à-dire, si $V$ est un champ de Killing. On en déduit que $H_{p}^{I}$ est définie négative sur le complémentaire orthogonal $C^{\prime}$ de $K$ dans $C$ et donc que

$$
\operatorname{Ind}_{p}(I) \geq \operatorname{dim} C^{\prime}=\operatorname{dim} C / K .
$$

On identifie, au moyen de la métrique $g$, les champs de vecteurs aux champs de 1-formes sur $M$ et on note $D^{*}$ l'adjoint de $D$ pour le produit scalaire $L^{2}$. La formule (5) peut alors s'écrire

$$
H_{p}^{I}(V, W)=m^{(p-2) / 2} \int_{M}\left\langle J_{p}^{I}(V), W\right\rangle d v
$$

où

$$
J_{p}^{I}(V)=D^{*} D V-\frac{(p-2)}{m} d \delta V-\operatorname{Ric}^{M} V .
$$

Le $p$-indice de $I$ est alors égal au nombre de valeurs propres (multiplicité comprise) strictement négatives de $J_{p}^{I}$. Si l'on note $\Delta_{H}=-(d \delta+\delta d)$ le laplacien de Hodge, on a alors (formule de Bochner-Weitzenböck) :

$$
D^{*} D=\Delta_{H}-\operatorname{Ric}^{M}
$$

et donc

$$
J_{p}^{I}=\Delta_{H}-\frac{(p-2)}{m} d \delta-2 \operatorname{Ric}^{M} .
$$

Dans le cas particulier où $(M, g)$ est d'Einstein, cette formule va nous permettre de relier, pour $p<m$, le $p$-indice de $I$ au spectre de $(M, g)$. En effet, pour tout $r>0$, on note $\lambda(r)$ le nombre de valeurs propres (multiplicité comprise) du Laplacien $\Delta$ de $(M, g)$ (agissant sur $C^{\infty}(M)$ ) strictement comprises entre 0 et $r$. On a alors le

THÉORÈME 3. - Soit $(M, g)$ une variété d'Einstein de dimension $m$ telle que $\mathrm{Ric}^{M}=c g$ avec $c>0$. Pour tout $p \in[2, m[$, on $a$

$$
\operatorname{Ind}_{p}(I)=\lambda\left(\frac{2 m c}{m+p-2}\right) .
$$


Preuve. - L'espace $\wedge^{1}(M)$ des 1-formes sur $M$ se décompose, par le théorème de Hodge de Rham, en la somme des deux sous-espaces orthogonaux $\operatorname{Im} d=\left\{d f ; f \in C^{\infty}(M)\right\}$ et $\operatorname{Ker} \delta=\left\{\alpha \in \wedge^{1}(M)\right.$; $\delta \alpha=0\}$. Dans le cas présent où la variété $(M, g)$ est d'Einstein, ces deux sous-espaces sont stables par l'opérateur $J_{p}^{I}=\Delta_{H}-\frac{(p-2)}{m} d \delta-2 c$ (vérification immédiate). Le spectre de $J_{p}^{I}$ est donc la réunion des spectres respectifs des opérateurs $J_{p}^{\prime}=J_{p}^{I} /$ Imd et $J_{p}^{\prime \prime}=J_{p}^{I} / \operatorname{Ker} \delta$. Or, la formule (6) nous dit que la forme $H_{p}^{I}$ est positive sur $\operatorname{Ker} \delta$, et donc que toutes les valeurs propres de $J_{p}^{\prime \prime}$ sont non-négatives. D'où

$$
\left.\operatorname{Ind}_{p}(I)=\# \operatorname{Spec}\left(J_{p}^{I}\right) \cap\right]-\infty, 0\left[=\# \operatorname{Spec}\left(J_{p}^{\prime}\right) \cap\right]-\infty, 0[.
$$

Pour toute forme $d f \in \operatorname{Im} d$, on a

$$
J_{p}^{\prime}(d f)=J_{p}^{I}(d f)=-d \delta d f-\frac{(p-2)}{m} d \delta d f-2 c d f
$$

D'où

$$
J_{p}^{\prime}(d f)=d\left(\frac{m+p-2}{m} \Delta f-2 c f\right) .
$$

On en déduit, sachant que $f$ peut toujours être choisie d'intégrale nulle sur $M$, que l'application

$$
\mu \mapsto \frac{m+p-2}{m} \mu-2 c
$$

est une bijection de $\operatorname{Spec}(\Delta) \backslash\{0\}$ sur $\operatorname{Spec}\left(J_{p}^{\prime}\right)$ (bijection qui conserve les multiplicités). En conclusion, on a

$$
\begin{aligned}
\operatorname{Ind}_{p}(I) & \left.=\# \operatorname{Spec}\left(J_{p}^{\prime}\right) \cap\right]-\infty, 0[ \\
& =\# \operatorname{Spec}(\Delta) \cap] 0, \frac{2 m c}{m+p-2}\left[=\lambda\left(\frac{2 m c}{m+p-2}\right)\right.
\end{aligned}
$$

Une conséquence immédiate du théorème 3 est le

COROLlaIRE 1. - Le p-indice de l'identité $I_{m}$ de la sphère canonique $\mathbb{S}^{m}$ est donné par

$$
\operatorname{Ind}_{p}\left(I_{m}\right)=\left\{\begin{array}{lll}
m+1 & \text { si } & p \in[2, m[ \\
0 & \text { si } & p \geq m .
\end{array}\right.
$$

En effet, sur $\mathbb{S}^{m}$, on a $c=m-1$ et l'intervalle $] 0, \frac{2 m(m-1)}{m+p-2}[$, contient pour $p<m$, une unique valeur propre de $\mathbb{S}^{m}, \lambda_{1}=m$, dont la multiplicité est $m+1$. 


\section{INDICE DES APPLICATIONS HOMOTHÉTIQUES}

Dans le cas où l'application $\phi$ est homothétique (i.e. $\phi^{*} g^{\prime}=k^{2} g$, où $k \in \mathbb{R})$, la $p$-tension $\tau_{p}(\phi)$, où $p \in[2,+\infty[$, est proportionnelle à la courbure moyenne de $\phi$. Par suite, l'application $\phi$ est $p$-harmonique si et seulement si elle est minimale. Or, une immersion minimale est un point critique de la fonctionnelle volume. À ce titre, elle possède un autre indice de Morse noté $\operatorname{Ind}_{V}(\phi)$.

THÉORÈME 4. - Soit $\phi$ une immersion homothétique minimale d'une variété riemannienne compacte $(M, g)$ dans une variété $\left(N, g^{\prime}\right)$. Pour tout $p \geq 2$ on $a$

$$
\operatorname{Ind}_{p}(\phi) \geq \operatorname{Ind}_{p}(I)
$$

où $I$ est l'identité de $M$. De plus, si $\phi$ est totalement géodésique (i.e. $\nabla^{\phi} d \phi=0$ ), alors on $a$

$$
\operatorname{Ind}_{p}(\phi)=\operatorname{Ind}_{p}(I)+\operatorname{Ind}_{V}(\phi) .
$$

Preuve. - Comme $\phi$ est homothétique, $|d \phi|$ est constante et on a (cf. (3)) pour tous $V, W \in \Gamma(\phi)$,

$$
\begin{aligned}
H_{p}^{\phi}(V, W)= & |d \phi|^{p-2} H_{2}^{\phi}(V, W)+(p-2)|d \phi|^{p-4} \\
& \times \int_{M}\left\langle\nabla^{\phi} V, d \phi(\cdot)\right\rangle\left\langle\nabla^{\phi} W, d \phi(\cdot)\right\rangle d v
\end{aligned}
$$

Notons $\Gamma^{T}(\phi)$ le sous-espace de $\Gamma(\phi)$ formé des champs $d \phi(X)$, où $X$ est un champ de vecteurs sur $M$. La restriction de $H_{2}^{\phi}$ à $\Gamma^{T}(\phi)$ est donnée par (voir par exemple [3], lemme 2.5) :

$$
H_{2}^{\phi}(d \phi(X), d \phi(X))=k^{2} H_{2}^{I}(X, X),
$$

où $k$ est la constante telle que $\phi^{*} g^{\prime}=k^{2} g$. De plus, on a

$$
\begin{aligned}
& \left\langle\nabla^{\phi} d \phi(X), d \phi(\cdot)\right\rangle=\left\langle\nabla^{\phi} d \phi(\cdot, X), d \phi(\cdot)\right\rangle+\langle d \phi(D . X), d \phi(\cdot)\rangle \\
& =0 \quad+k^{2}\langle D . X, \cdot\rangle,
\end{aligned}
$$

car, comme $\phi$ est homothétique, la forme $\nabla^{\phi} d \phi$ prend ses valeurs dans le fibré normal de $\phi$. Le report de (11) et (12) dans (10) donne

$$
H_{p}^{\phi}(d \phi(X), d \phi(Y))=k^{2} H_{p}^{I}(X, Y) .
$$

Vol. $13, \mathrm{n}^{\circ} 2-1996$ 
On en déduit immédiatement la première insertion du théorème.

Supposons maintenant $\phi$ totalement géodésique et notons $\Gamma^{N}(\phi)$ l'espace des champs normaux le long de $\phi$. On a alors $\Gamma(\phi)=\Gamma^{T}(\phi) \oplus \Gamma^{N}(\phi)$. Nous allons montrer que, pour tout $d \phi(X) \in \Gamma^{T}(\phi)$ et tout $V \in \Gamma^{N}(\phi)$, on a

$$
H_{p}^{\phi}(d \phi(X), V)=0
$$

En effet, comme $\phi$ est totalement géodésique, on a, pour tous $X, Y, Z$ champs de vecteurs sur $M$,

$$
\begin{gathered}
\nabla_{Y}^{\phi} d \phi(X)=d \phi\left(D_{Y} X\right) \\
\left\langle\nabla_{Y}^{\phi} V, d \phi(Z)\right\rangle=-\left\langle V, \nabla_{Y}^{\phi} d \phi(X)\right\rangle=0
\end{gathered}
$$

et donc par conséquence,

$$
\left\langle\nabla^{\phi} d \phi(X), \nabla^{\phi} V\right\rangle=0 .
$$

Par ailleurs, la formule de Weitzenböck [1] donne dans le cas totalement géodésique

$$
\operatorname{Ric}^{\phi}(d \phi(X), V)=\left\langle d \phi\left(\operatorname{Ric}^{M} X\right), V\right\rangle=0 .
$$

Le report de (15), (16) et (17) dans (3) donne (14). La propriété d'orthogonalité pour $H_{p}^{\phi}$ qu'exprime (14) entraîne :

$$
\operatorname{Ind}_{p}(\phi)=\operatorname{Ind}\left(H_{p}^{T}\right)+\operatorname{Ind}\left(H_{p}^{N}\right),
$$

où Ind $\left(H_{p}^{T}\right)$ (resp. Ind $\left.\left(H_{p}^{N}\right)\right)$ est l'indice de la restriction $H_{p}^{T}\left(\right.$ resp. $\left.H_{p}^{N}\right)$ de la forme $H_{p}^{\phi}$ à $\Gamma^{T}(\phi)\left(\right.$ resp. $\left.\Gamma^{N}(\phi)\right)$. Or, on vient de voir (d'après (13)) que

$$
\operatorname{Ind}\left(H_{p}^{T}\right)=\operatorname{Ind}_{\mathrm{p}}(I) \text {. }
$$

D'autre part, la forme $H_{p}^{N}=|d \phi|^{p-2} H_{2}^{\phi} / \Gamma^{N}(d \phi)$ est proportionnelle, dans le cas totalement géodésique, à la variation seconde du volume en $\phi(c f .[10])$. D'où

$$
\operatorname{Ind}\left(H_{p}^{N}\right)=\operatorname{Ind}_{\mathrm{V}}(\phi)
$$

La seconde assertion découle alors de (18), (19) et (20). 
Notons $I_{m, n}$ l'injection canonique de $\mathbb{S}^{m}$ dans $\mathbb{S}^{n}$, où $m \leq n$. Simons montre dans [10] que $\operatorname{Ind}_{V}\left(I_{m, n}\right)=n-m$. La combinaison de ce résultat avec ceux du théorème 4 et du corollaire 1 donne le

Corollaire 2. - On a

$$
\operatorname{Ind}_{p}\left(I_{m, n}\right)=\left\{\begin{array}{lll}
n+1 & \text { si } & p \in[2, m[ \\
n-m & \text { si } & p \geq m .
\end{array}\right.
$$

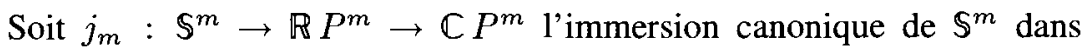
l'espace projectif complexe $\subset P^{m}$ de dimension réelle $2 m$. Cette immersion est homothétique et totalement géodésique et on a ( $c f .[3])$

$$
\operatorname{Ind}_{V}\left(j_{m}\right)=\frac{(m+1)(m+2)}{2} .
$$

D'où le

Corollaire 3. - On a

$$
\operatorname{Ind}_{p}\left(j_{m}\right)=\left\{\begin{array}{lll}
(m+1)(m+4) / 2 & \text { si } & p \in[2, m[ \\
(m+1)(m+2) / 2 & \text { si } & p \geq m
\end{array}\right.
$$

\section{APPLICATIONS $p$-HARMONIQUES DE LA SPHÈRE}

Soit $G(m)$ le groupe des difféomorphismes conformes de $\mathbb{S}^{m}$. L'espace $C$ des champs conformes de $\mathbb{S}^{m}$ est la somme directe de l'espace $K$ des champs de Killing et de l'espace $A$ des champs gradients des premières harmoniques sphériques : pour tout $a \in R^{m+1}$, on note $\bar{a}$ le gradient dans $\mathbb{S}^{m}$ de la fonction $x \rightarrow\langle x, a\rangle$ (i.e. $\left.\bar{a}(x)=a-\langle x, a\rangle x\right)$, on a alors

$$
A=\left\{\vec{a} ; a \in \mathbb{R}^{m+1}\right\} .
$$

Soit $\phi$ une application $p$-harmonique de $\mathbb{S}^{m}$ dans une variété riemannienne $\left(N, g^{\prime}\right)$. Dans ce paragraphe, nous allons nous intéresser à la restriction de la $p$-énergie $E_{p}$ à la sous-variété $G_{m}(\phi)=\{\phi \circ \gamma ; \gamma \in G(m)\}$ dont l'espace tangent en $\phi$ est $d \phi(C)=d \phi(K) \oplus d \phi(A) \subset \Gamma(\phi)$.

Le premier résultat que nous obtenons concerne la dérivée seconde de cette fonction. Comme $d \phi(K)$ fait partie du noyau de la variation seconde $H_{p}^{\phi}$ de $E_{p}$, il suffit de déterminer la restriction de $H_{p}^{\phi}$ à $d \phi(A)$. Celle-ci est donnée par le 
ThÉORÈME 5. - Soit $\phi$ une application p-harmonique non constante de $\mathbb{S}^{m}$ dans une variété $\left(N, g^{\prime}\right)$. Pour tout $a \in \mathbb{R}^{m+1}$ on a

$$
H_{p}^{\phi}(d \phi(\bar{a}), d \phi(\bar{a}))=\frac{p-m}{|a|} \int_{M}|d \phi|^{p-2}|d \phi(\bar{a})|^{2} d v .
$$

En particulier,

(i) si $p<m$, alors $H_{p}^{\phi}$ est définie négative sur $d \phi(A)$ et on a

$$
\operatorname{Ind}_{p}(\phi) \geq m+1
$$

(ii) si $p<m$, alors $H_{p}^{\phi}$ est définie positive sur $d \phi(A)$.

Il découle de ce théorème que, si $p<m$ (resp. $p>m$ ), alors la restriction de $E_{p}$ à $G_{m}(\phi)$ admet un maximum local (resp. minimum local) en $\phi$. Le résultat suivant nous dit que ce maximum (resp. ce minimum) est en fait global.

THÉORÈME 6. - Soit $\phi$ une application p-harmonique non constante de $\mathbb{S}^{m}$ dans une variété $\left(N, g^{\prime}\right)$.

(i) Si $p<m$, alors on a

$$
E_{p}(\phi)=\operatorname{Sup}_{\gamma \in G(m)} E_{p}(\phi \circ \gamma)
$$

De plus, l'égalité $E_{p}(\phi)=E_{p}(\phi \circ \gamma)$ n'a lieu que si $\gamma$ est une isométrie.

(ii) Si $p>m$, alors on $a$

$$
E_{p}(\phi)=\operatorname{Inf}_{\gamma \in G(m)} E_{p}(\phi \circ \gamma)
$$

De plus, l'égalité $E_{p}(\phi)=E_{p}(\phi \circ \gamma)$ n'a lieu que si $\gamma$ est une isométrie.

Pour $p=m, E_{m}$ est constant sur $G_{m}(\phi)$ (ceci peut se voir comme application de la formule de changement de variable).

La preuve de ces théorèmes nécessite les lemmes suivants :

Lemme 1. - Si $\gamma$ est un difféomorphisme conforme d'une variété riemannienne $(M, g)$, alors pour tout $p \geq 2$, on $a$

$$
\tau_{p}(\gamma)=m^{(p-2) / 2}(p-m) \alpha^{p-3} d \gamma(\nabla \alpha)
$$

où $\alpha$ est la fonction telle que $\gamma^{*} g=\alpha^{2} g$. 
Preuve. - Comme $\gamma$ est conforme on a $|d \gamma|^{2}=m \alpha^{2}$ et donc (cf. (1))

$$
\begin{aligned}
\tau_{p}(\gamma) & =|d \gamma|^{p-2} \tau_{2}(\gamma)+d \gamma\left(\nabla|d \gamma|^{p-2}\right) \\
& =m^{(p-2) / 2}\left(\alpha^{p-2} \tau_{2}(\gamma)+d \gamma\left(\nabla \alpha^{p-2}\right)\right. \\
& =m^{(p-2) / 2} \alpha^{p-3}\left(\alpha \tau_{2}(\gamma)+(p-2) d \gamma(\nabla \alpha)\right) .
\end{aligned}
$$

Si l'on note $\tilde{D}$ la connexion de Levi-Civita de $\tilde{g}=\alpha^{2} g$, alors on a

$$
\nabla_{X}^{\gamma} d \gamma(Y)=d \gamma\left(\tilde{D}_{X} Y\right)
$$

(car $\gamma$ est un difféomorphisme isométrique de $(M, \tilde{g})$ sur $(M, g)$ ). On a donc

$$
\left(\nabla^{\gamma} d \gamma\right)(X, Y)=d \gamma\left(\tilde{D}_{X} Y-D_{X} Y\right)
$$

et, dans un repère orthonormé local $\left\{e_{i}\right\}_{i}$,

Or, on a

$$
\tau_{2}(\gamma)=\sum_{i} d \gamma\left(\tilde{D}_{e_{i}} e_{i}-D_{e_{i}} e_{i}\right)
$$

$$
\tilde{D}_{X} Y-D_{X} Y=\alpha^{-1}(\langle X, \nabla \alpha\rangle Y+\langle Y, \nabla \alpha\rangle X-\langle X, Y\rangle \nabla \alpha) .
$$

On en déduit

$$
\tau_{2}(\alpha)=(2-m) \alpha^{-1} d \gamma(\nabla \alpha)
$$

D'où le lemme 1.

LEMME 2. - Si $(M, g),\left(N, g^{\prime}\right)$ et $\left(P, g^{\prime \prime}\right)$ sont trois variétés riemanniennes et si $\phi: M \rightarrow N$ et $\psi: N \rightarrow P$ sont des applications différentiables, alors

$$
\begin{aligned}
\tau_{p}(\psi \circ \phi)= & f d \psi\left(\tau_{p}(\phi)\right)+|d(\psi \circ \phi)|^{p-2} \operatorname{tr}_{g} \nabla^{\psi} d \psi(d \phi(\cdot), d \phi(\cdot)) \\
& +|d \phi|^{p-2} d(\psi \circ \phi)(\nabla f)
\end{aligned}
$$

où $f=|d(\psi \circ \phi)|^{p-2} /|d \phi|^{p-2}$.

Ce lemme s'obtient sans difficulté à partir de (2) et de l'expression de $\tau_{2}(\psi \circ \phi)$ donnée dans [1].

Une application des deux lemmes précédents est le

LEMME 3. - Soient $(M, g)$ et $\left(N, g^{\prime}\right)$ deux variétés riemanniennes, $\phi: M \rightarrow N$ une application différentiable et $\gamma$ un difféomorphisme conforme de $(M, g)$. Pour tout $p \geq 2$ on a

$$
\tau_{p}(\phi \circ \gamma)=(p-m) \alpha^{p-3}|d \phi|^{p-2} d(\phi \circ \gamma)(\nabla \alpha)+\alpha^{p} \tau_{p}(\phi) \circ \gamma
$$

où $\alpha$ est telle que $\gamma^{*} g=\alpha^{2} g$.

Vol. $13, n^{\circ} 2-1996$ 
Preuve. - Si $\left\{e_{i}\right\}_{i}$ est un repère orthonormé local au voisinage d'un point $x \in M$, alors $\left\{\alpha^{-1} d \gamma\left(e_{i}\right)\right\}_{i}$ est un repère orthonormé local au voisinage de $\gamma(x)$. On a donc (lemme 2 ),

$$
\begin{aligned}
& |d(\phi \circ \gamma)|^{2}=\alpha^{2}|d \phi|^{2} \circ \gamma \\
& f=m^{(2-p) / 2}|d \phi|^{p-2} \circ \gamma \\
& \operatorname{tr}_{g} \nabla^{\phi} d \phi(d \gamma(\cdot), d \gamma(\cdot))=\alpha^{2} \tau_{2}(\phi),
\end{aligned}
$$

et

$$
\tau_{p}(\phi \circ \gamma)=m^{(2-p) / 2}|d \phi|^{p-2} \circ \gamma d \phi\left(\tau_{p}(\gamma)\right)+\alpha^{p} \tau_{p}(\phi) \circ \gamma
$$

Le report de la formule du lemme 1 dans (21) donne le résultat.

Pour tout $a \in \mathbb{R}^{m+1}$, on désigne par $\left(\gamma_{t}^{a}\right)_{t}$ le groupe à 1 paramètre de difféomorphismes conformes engendré par le champ $\bar{a}$ sur $\mathbb{S}^{m}$. On note $\alpha_{t}^{a}$ la fonction telle que $\left(\gamma_{t}^{a}\right)^{*}$ can $=\left(\alpha_{t}^{a}\right)^{2}$ can, où can est la métrique canonique de $\mathbb{S}^{m}$. D'après [5], on a

$$
\alpha_{t}^{a}(x)=|a|(\operatorname{sh} t\langle x, a\rangle+|a| \operatorname{ch} t)^{-1} .
$$

LEMME 4. - Soit $\phi$ une application $p$-harmonique $(p \geq 2)$ de $\mathbb{S}^{m}$ dans une variété riemannienne $\left(N, g^{\prime}\right)$. Pour tout a $\in \mathbb{R}^{m+1} \backslash\{0\}$ et tout $t_{0} \in R$, on a

$$
\begin{aligned}
& \frac{d}{d t} E_{p}\left(\left.\phi \circ \gamma_{t}^{a}\right|_{t=t_{0}}\right. \\
& \quad=\frac{(p-m)}{|a|} \operatorname{sh} t_{0} \int_{S^{m}}|d \phi|^{p-2} \circ \gamma_{t_{0}}^{a}\left(\alpha_{t_{0}}^{a}\right)^{p-1}\left|d\left(\phi \circ \gamma_{t_{0}}^{a}\right)(\bar{a})\right|^{2} d v .
\end{aligned}
$$

Preuve. - La formule de la variation première (2) donne :

$$
\begin{aligned}
\left.\frac{d}{d t} E_{p}\left(\phi \circ \gamma_{t}^{a}\right)\right|_{t=t_{0}} & =\left.\frac{d}{d t} E_{p}\left(\phi \circ \gamma_{t_{0}+t}^{a}\right)\right|_{t=0} \\
& =-\int_{S^{m}}\left\langle\tau_{p}\left(\phi \circ \gamma_{t_{0}}^{a}\right), d\left(\phi \circ \gamma_{t_{0}}^{a}\right)(\bar{a})\right\rangle d v
\end{aligned}
$$

$\left.\operatorname{car} \frac{d}{d t} \phi \circ \gamma_{t_{0}+t}^{a}\right|_{t=t_{0}}=\left.\frac{d}{d t}\left(\phi \circ \gamma_{t_{0}}^{a}\right) \circ \gamma_{t}^{a}\right|_{t=0}=d\left(\phi \circ \gamma_{t_{0}}^{a}\right)(\bar{a})$

Or, on a

$$
\nabla \alpha_{t}^{a}=-\frac{\operatorname{sh} t}{|a|}\left(\alpha_{t}^{a}\right)^{2} \bar{a}
$$

Le lemme 3 nous donne donc, avec $\tau_{p}(\phi)=0$,

$$
\tau_{p}\left(\phi \circ \gamma_{t}^{a}\right)=\frac{(p-m)}{|a|} \operatorname{sh} t\left(\alpha_{t}^{a}\right)^{p-1}|d \phi|^{p-2} \circ \gamma_{t}^{a} d\left(\phi \circ \gamma_{t}^{a}\right)(\bar{a}) \text {. }
$$


le résultat en découle immédiatement.

Preuve du théorème 5. - On a

$$
H_{p}^{\phi}\left(d \phi(\bar{a}), d \phi(\bar{a})=\left.\frac{d^{2}}{d t^{2}} E_{p}\left(\phi \circ \gamma_{t}^{a}\right)\right|_{t=0} .\right.
$$

Il suffit donc de dériver en $t_{0}=0$ le membre de droite de la formule du lemme 4. La présence de $\operatorname{sh} t_{0}$ rend ce calcul immédiat :

$$
H_{p}^{\phi}(d \phi(\bar{a}), d \phi(\bar{a}))=(p-m)|a|^{-1} \int_{M}|d \phi|^{p-2}|d \phi(\bar{a})|^{2} v .
$$

Il est clair que si $p<m$ (resp. $p>m$ ), alors $H_{p}^{\phi}$ est définie négative (resp. définie positive) sur $d \phi(A)$. Pour $p<m$ on a donc

$$
\operatorname{Ind}_{p}(\phi) \geq \operatorname{dim} d \phi(A)
$$

Or, d'après [3], on sait que, pour toute application $\phi$ non constante, on a $\operatorname{dim} d \phi(A)=\operatorname{dim} A=m+1$.

Preuve du théorème 6. - Dans la formule du lemme 4, le membre de droite ne s'annule que si $t_{0}=0$ ou si $d\left(\phi \circ \gamma_{t_{0}}^{a}\right)(\bar{a})=0$. Mais, d'après [3], cette dernière condition entraîne, pour $a \neq 0$, que $\phi \circ \gamma_{t_{0}}^{a}$ (et donc $\phi$ ) est constante. Comme $\phi$ est non constante on en déduit que, si $p<m$ (resp. $p<m$ ), alors, pour tout $a \in \mathbb{R}^{m+1} \backslash\{0\}$, la fonction $t \mapsto E_{p}\left(\phi \circ \gamma_{t}^{a}\right)$ est strictement décroissante (resp. strictement croissante) sur l'intervalle $[0,+\infty[$ et on a, pour tout $t>0$,

$$
E_{p}\left(\phi \circ \gamma_{t}^{a}\right)<E_{p}(\phi)
$$

(resp. $\left.E_{p}\left(\phi \circ \gamma_{t}^{a}\right)>E_{p}(\phi)\right)$. Or, on a (cf. [4])

$$
G(m)=\left\{r \gamma_{t}^{a} ; r \in 0(m+1), a \in S^{m}, t \in[0,+\infty[\} .\right.
$$

On en déduit que, si $p<m$ (resp. $p>m$ ), alors, pour tout $\gamma=r \gamma_{t}^{a} \in$ $G(m)$, on a

$$
E_{p}(\phi \circ \gamma)=E_{p}\left(\phi \circ r \circ \gamma_{t}^{a}\right) \leq E_{p}(\phi \circ r)=E_{p}(\phi)
$$

(resp. $\left.E_{p}(\phi \circ \gamma) \geq E_{p}(\phi)\right)$. De plus, l'égalité $E_{p}(\phi \circ \gamma)=E_{p}(\phi)$ n'a lieu que si $t=0$, i.e., si $\gamma=r \in 0(m+1)$.

Remarque. - On peut vérifier très facilement que le théorème 6 reste valable sous l'hypothèse plus faible suivante: en tout point $x$ de $\mathbb{S}^{m}, \tau_{p}(\phi)(x)$ est orthogonal à $d \phi\left(T_{x} \mathbb{S}^{m}\right)$. Noter que cette hypothèse est vérifiée en particulier par toutes les immersions homothétiques (dans ce cas $\tau_{p}(\phi)$ est proportionnel à la courbure moyenne). 


\section{APPLICATIONS $p$-HARMONIQUES A VALEURS DANS $S^{n}$}

Soit $\phi$ une application $p$-harmonique d'une variété compacte $(M, g)$ dans $\mathbb{S}^{n}$. Dans ce paragraphe, nous nous intéressons à la restriction de la $p$-énergie à la sous-variété $G_{n}(\phi)=\{\gamma \circ \phi ; \gamma \in G(n)\}$ dont l'espace tangent en $\phi$ est $C_{\phi}=K_{\phi} \oplus A_{\phi} \subset \Gamma(\phi)$ où $K_{\phi}=\{X \circ \phi ; X \in K\}$ et $A_{\phi}=\left\{\bar{a} \circ \phi ; a \in \mathbb{R}^{n+1}\right\}$ (les notations sont celles du paragraphe précédent). Là aussi, le sous-espace $K_{\phi}$ fait partie du noyau de $H_{p}^{\phi}$. La restriction de $H_{p}^{\phi}$ à $A_{\phi}$ est donnée par la

Proposition 4. - Soit $\phi$ une application p-harmonique non constante $(p \geq 2)$ d'une variété riemannienne compacte $(M, g)$ dans $\mathbb{S}^{m}$. Pour tout $a \in \mathbb{R}^{n+1}$ on $a$

$$
H_{p}^{\phi}(\bar{a} \circ \phi)=\int_{M}|d \phi|^{p-2}\left(p\left|d \phi_{a}\right|^{2}-|d \phi|^{2}|\bar{a} \circ \phi|^{2}\right) d v,
$$

où $\phi_{a}=\langle\phi, a\rangle$. En particulier, si $\phi(M)$ est contenue dans une sphère totalement géodésique de dimension $k$, alors,

$$
\operatorname{Ind}_{p}(\phi) \geq n-k \text {. }
$$

Par un raisonnement identique à celui utilisé dans [2], théorème 2.4, nous en déduisons le

THÉORÈME 7. - Toute application p-harmonique non constante $(p \geq 2)$ d'une variété compacte $(M, g)$ dans $\mathbb{S}^{n}$ vérifie

$$
\operatorname{Ind}_{p}(\phi) \geq n-p \text {. }
$$

De plus, si l'égalité a lieu, alors $\phi(M)$ est contenue dans une sphère totalement géodésique de dimension $p$.

L'optimalité de cette minoration découle du corollaire 2.

Pour avoir des résultats du même type que ceux du précédent paragraphe, nous sommes amenés à nous restreindre au cas des applications dont le tenseur de $p$-énergie-impulsion est positif. Ce tenseur, noté $S_{p}(\phi)$, est donné par

$$
S_{p}(\phi)=\frac{1}{p}|d \phi|^{p} g-|d \phi|^{p-2} \phi^{*} g^{\prime} .
$$

Pour tout $x \in M$, on pose

$$
S_{p}^{0}(\phi)(x)=\operatorname{Inf}\left\{S_{p}(\phi)(X, X) ; X \in T_{x} M \text { et }|X|=1\right\} .
$$

Le tenseur $S_{p}(\phi)$ est dit positif (resp. défini positif) au point $x$ si $S_{p}^{0}(\phi)(x) \geq 0\left(\operatorname{resp} . S_{p}^{0}(\phi)(x)>0\right)$. 
Notons que :

(i) $\operatorname{tr}_{g} S_{p}(\phi)=(m-p)|d \phi|^{p} / p$. Par suite, si $p \geq m$, alors $S_{p}(\phi)$ n'est jamais défini positif.

(ii) Si $\phi$ est homothétique, alors $S_{p}(\phi)=\left(\frac{1}{p}-\frac{1}{m}\right)|d \phi|^{p}$ est défini positif pour tout $p<m$.

(iii) Si $\phi$ est horizontalement conforme (cf. [1]), alors on a $S_{p}^{0}(\phi)=$ $\left(\frac{1}{p}-\frac{1}{n}\right)|d \phi|^{p}$ et $S_{p}(\phi)$ est défini positif si et seulement si $p<n$.

THÉOREME 8. - Soit $\phi$ une application p-harmonique $(p \geq 2)$ d'une variété riemannienne compacte $(M, g)$ dans $\mathbb{S}^{n}$. Si le tenseur de p-énergieimpulsion de $\phi$ est défini positif sur $M$, alors $H_{p}^{\phi}$ est définie négative sur $A_{\phi}$ et on a

$$
\operatorname{Ind}_{p}(\phi) \geq n+1 \text {. }
$$

Il découle de ce théorème que, si $S_{p}(\phi)$ est défini positif, alors la restriction de $E_{p}$ à $G_{n}(\phi)$ admet un maximum local en $\phi$. Le résultat suivant nous dira que ce maximum est en fait global.

THÉORÈME 9. - Soit $\phi$ une application p-harmonique $(p \geq 2)$ d'une variété riemannienne compacte $(M, g)$ dans $\mathbb{S}^{n}$. Si le tenseur de p-énergieimpulsion $S_{p}(\phi)$ de $\phi$ est positif sur $M$, alors

$$
E_{p}(\phi)=\operatorname{Sup}_{\gamma \in G(n)} E_{p}(\gamma \circ \phi)
$$

De plus, si $S_{p}(\phi)$ est défini positif, alors l'égalité $E_{p}(\gamma \circ \phi)=E_{p}(\phi) n^{\prime} a$ lieu que si $\gamma$ est une isométrie.

La preuve de ces résultats nécessite le lemme suivant :

LEMME 5. - Soient $(M, g)$ et $\left(N, g^{\prime}\right)$ deux variétés riemanniennes, $\phi$ une application différentiable de $M$ dans $N$ et $\gamma$ un difféomorphisme conforme de $\left(N, g^{\prime}\right)$. Pour tout $p \geq 2$ on a

$$
\begin{aligned}
\tau_{p}(\gamma \circ \phi)= & \alpha^{p-2} \circ \phi d \gamma\left(\tau_{p}(\phi)\right) \\
& +\alpha^{p-3} \circ \phi|d \phi|^{p-2} d \gamma\left(p d \phi(\nabla(\alpha \circ \phi))-|d \phi|^{2} \nabla \alpha \circ \phi\right)
\end{aligned}
$$

où $\alpha$ telle que $\gamma^{*} g^{\prime}=\alpha^{2} g^{\prime}$.

Preuve. - On a, dans un repère orthonormé local $\left\{e_{i}\right\}_{i}$ de $M$ :

$$
|d(\gamma \circ \phi)|^{2}=\sum_{i}\left|d \gamma d \phi\left(e_{i}\right)\right|^{2}=\alpha^{2} \circ \phi|d \phi|^{2}
$$


et ( $c f$. preuve du lemme 1)

(23) $\operatorname{tr}_{g} \nabla^{\gamma} d \gamma(d \phi(\cdot), d \phi(\cdot))$

$$
\begin{aligned}
& =\alpha^{-1} \circ \phi d \gamma\left(2 \sum_{i}\left\langle d \phi\left(e_{i}\right),(\nabla \alpha) \circ \phi\right\rangle d \phi\left(e_{i}\right)-\left|d \phi\left(e_{i}\right)\right|^{2}(\nabla \alpha) \circ \phi\right) \\
& =\alpha^{-1} \circ \phi d \gamma\left(2 d \phi(\nabla(\alpha \circ \phi))-|d \phi|^{2}(\nabla \alpha) \circ \phi\right) .
\end{aligned}
$$

Le report de (22) et (23) dans la formule du lemme 2 donne le résultat.

Avec les notations du paragraphe précédent on a :

LEMME 6. - Soit $\phi$ une application p-harmonique d'une variété compacte $(M, g)$ dans $S^{n}$. Pour tout $a \in \mathbb{R}^{n+1}$ et tout $t_{0} \in R$ on $a$ :

$$
\begin{aligned}
& \left.\frac{d}{d t} E_{p}\left(\gamma_{t}^{a} \circ \phi\right)\right|_{t=t_{0}} \\
& \quad=\frac{\operatorname{sh} t_{0}}{|a|} \int_{M}\left(\alpha_{t_{0}}^{a} \circ \phi\right)^{p+1}|d \phi|^{p-2}\left(p\left|d \phi_{a}\right|^{2}-|d \phi|^{2}|\bar{a} \circ \phi|^{2}\right) d v
\end{aligned}
$$

Preuve. - La formule de la variation première donne :

$$
\begin{aligned}
\left.\frac{d}{d t} E_{p}\left(\gamma_{t}^{a} \circ \phi\right)\right|_{t=t_{0}} & =\left.\frac{d}{d t} E_{p}\left(\gamma_{t_{0}+t}^{a} \circ \phi\right)\right|_{t=0} \\
& =-\int_{M}\left\langle\tau_{p}\left(\gamma_{t_{0}}^{a} \circ \phi\right), d \gamma_{t_{0}}^{a}(\bar{a} \circ \phi)\right\rangle d v
\end{aligned}
$$

car

$$
\left.\frac{d}{d t} \gamma_{t_{0}+t}^{a} \circ \phi\right|_{t=0}=\frac{d}{d t} \gamma_{t_{0}}^{a}\left(\gamma_{t}^{a} \circ \phi\right)=d \gamma_{t_{0}}^{a}(\bar{a} \circ \phi)
$$

D'après le lemme 5 , et comme $\tau_{p}(\phi)=0$, on a

$$
\begin{aligned}
& \left\langle\tau_{p}\left(\gamma_{t}^{a} \circ \phi\right), d \gamma_{t}^{a}(\bar{a} \circ \phi)\right\rangle \\
& \quad=\left(\alpha_{t}^{a} \circ \phi\right)^{p-1}\left\langle p d \phi\left(\nabla\left(\alpha_{t}^{a} \circ \phi\right)\right)-|d \phi|^{2}\left(\nabla \alpha_{t}^{a}\right) \circ \phi, \bar{a} \circ \phi\right\rangle
\end{aligned}
$$

Or, on a

$$
\left\langle\left(\nabla \alpha_{t}^{a}\right) \circ \phi, \bar{a} \circ \phi\right\rangle=-\frac{\operatorname{sh} t}{|a|}\left(\alpha_{t}^{a} \circ \phi\right)^{2}|\bar{a} \circ \phi|^{2}
$$


et, dans un repère orthonormé local $\left\{e_{i}\right\}_{i}$ de $M$,

$$
\begin{aligned}
\left.\left\langle\nabla\left(\alpha_{t}^{a}\right) \circ \phi\right), \bar{a} \circ \phi\right\rangle & \left.=\sum_{i}\left\langle\left\langle\nabla \alpha_{t}^{a}\right) \circ \phi, d \phi\left(e_{i}\right)\right\rangle d \phi\left(e_{i}\right), \bar{a} \circ \phi\right\rangle \\
& =-\frac{\operatorname{sh} t}{|a|}\left(\alpha_{t}^{a} \circ \phi\right)^{2} \sum_{i}\left\langle\bar{a} \circ \phi, d \phi\left(e_{i}\right)\right\rangle^{2} \\
& =-\frac{\operatorname{sh} t}{|a|}\left(\alpha_{t}^{a} \circ \phi\right)^{2}\left|d \phi_{a}\right|^{2} .
\end{aligned}
$$

Le report de (25) et (26) dans (24) donne le résultat.

Preuve de la proposition 4. - En dérivant en $t_{0}=0$ la formule du lemme 6 on obtient

$$
\begin{aligned}
H_{p}^{\phi}(\bar{a} \circ \phi, \bar{a} \circ \phi) & =\left.\frac{d^{2}}{d t^{2}} E_{p}\left(\gamma_{t}^{a} \circ \phi\right)\right|_{t=0} \\
& =\int_{M}|d \phi|^{p-2}\left(p\left|d \phi_{a}\right|^{2}-|d \phi|^{2}|\bar{a} \circ \phi|^{2}\right) d v
\end{aligned}
$$

Maintenant, si $\phi(M)$ est contenue dans une $k$-sphère, alors il existe un sous-espace $E$ de dimension $k+1$ de $R^{n+1}$ tel qu'on ait $\phi(M) \subset E$. Pour tout $a \in E^{\perp}$, complémentaire orthogonal de $E$, on a $\phi_{a}=0$ et donc

$$
H_{p}^{\phi}(\bar{a} \circ \phi, \bar{a} \circ \phi)=-\int_{M}|d \phi|^{p}|\bar{a} \circ \phi|^{2} d v .
$$

D'où, $H_{p}^{\phi}$ est définie négative sur le sous-espace $\varepsilon=\left\{\bar{a} \circ \phi ; a \in E^{\perp}\right\}$. Comme $\phi$ est non constante on a $\operatorname{dim} \varepsilon=\operatorname{dim} E^{\perp}=n-k$ (en effet, l'application linéaire $a \mapsto \bar{a} \circ \phi$ de $\mathbb{R}^{n+1}$ dans $\Gamma(\phi)$ est injective car $\bar{a}$ ne s'annule sur $\mathbb{S}^{n}$ qu'aux points $\left.\pm a /|a|\right)$. Par suite, on a

$$
\operatorname{Ind}_{p}(\phi) \geq \operatorname{dim} \varepsilon=n-k .
$$

Preuve du théorème 8 . - Par un raisonnement identique à celui utilisé dans la preuve du théorème 2.5 de [2] on peut montrer que

$$
|d \phi|^{p-2}\left(p\left|d \phi_{a}\right|^{2}-|d \phi|^{2}|\bar{a} \circ \phi|^{2}\right) \leq-p S_{p}^{0}(\phi)|\bar{a} \circ \phi|^{2} .
$$

Le report de (27) dans la formule de la proposition 3 montre que, sous l'hypothèse « $S_{p}(\phi)$ défini positif», $H_{p}^{\phi}$ est définie négative sur le sous-espace $A_{\phi}$ et donc qu'on a

$$
\operatorname{Ind}_{p}(\phi) \geq \operatorname{dim} A_{\phi}=n+1 .
$$

Vol. 13, $n^{\circ} 2-1996$. 
Preuve du théorème 9. - Le report de (27) dans la formule du lemme 6 montre que, sous l'hypothèse « $S_{p}(\phi)$ positif» (resp. « $S_{p}(\phi)$ défini positif ») la fonction $t \mapsto E_{p}\left(\gamma_{t}^{a} \circ \phi\right)$ est décroissante (resp. strictement décroissante) sur $\left[0,+\infty\left[\right.\right.$ et donc qu'on a, pour tout $a \bar{\epsilon} R^{n+1} \backslash\{0\}$ et tout $t>0$,

$$
\left.E_{p}\left(\gamma_{t}^{a} \circ \phi\right) \leq E_{p}(\phi) \quad \text { (resp. } E_{p}\left(\gamma_{t}^{a} \circ \phi\right)<E_{p}(\phi)\right) .
$$

Ceci suffit pour montrer le théorème 9 ( $c f$. preuve du théorème 6 ).

\section{RÉFÉRENCES}

[1] J. Eells et L. Lemaire, Selected topics in harmonic maps, C.B.M.S. Regional Conf. Ser. 50, A.M.S., Providence, 1983.

[2] A. El Souf,, Applications harmoniques, immersions minimales et transformations conformes de la sphère, Compositio Math., vol. 85, 1993, p. 281-298.

[3] A. El Soufr, Indice de Morse des Applications harmoniques de la sphère, Compositio Math., vol. 95, 1995, p. 343-362.

[4] A. El Soufi et S. ILIAS, Immersions minimales, première valeur propre du Laplacien et volume conforme, Math. Ann., vol. 275, 1986, p. 257-267.

[5] A. El Soufl et S. ILIAS, Une inégalité du type « Reilly » pour les sous-variétés de l'espace hyperbolique, Comment. Math. Helv., vol. 67, 1992, p. 167-181.

[6] A. El Soufi et A. Jeune, Indice de Morse des Applications p-harmoniques, C. R. Acad. Sci. Paris, t. 315, série I, 1992, p. 1189-1192.

[7] A. Jeune, Thèse, Université de Tours, 1993.

[8] P. F. Leung, On the stability of harmonic maps, Lecture Notes in Math. $n^{\circ} 949,1982$, p. $122-129$.

[9] E. Mazet, La formule de la variation seconde de l'énergie etc., J. Diff. Geom., vol. 8, 1973, p. 279-296.

[10] J. Simons, Minimal varieties in Riemannian manifolds, Ann. of Math., vol. 88, 1968, p. 62-105.

[11] R. T. Sмітн, The second variation formula for harmonic mappings, Proc. Amer. Math. Soc., vol. 47, 1975, p. 229-236.

[12] K. Uhlenbeck, Minimal Spheres and other conformal Variationnal problems, Seminar on Minimal Submanifolds, Princeton University press, 1983, p. 169-176.

[13] Y. L. XIN, Some results on stable harmonic maps, Duke Math. J., vol. 47, 1980, p. 609-613.

[14] K. Yano et S. Bochner, Curvature and Betti numbers, Ann. of Math. Studies, $\mathrm{n}^{\circ} 32$, Princeton Univ. press, 1953.

(Manuscrit rę̧u le 16 juin 1994; version révisée reçue le 23 janvier 1995.) 